\title{
Analysis of Spices Available in Local Market in Bangladesh Using Ion Beam Analysis Technique
}

\author{
Shirin Akter ${ }^{1 *}$, Rajada Khatun ${ }^{2}$, M. Monjur Ahasan ${ }^{1}$, Md. Joynal Abedin ${ }^{1}$, \\ Md. Fakhar Uddin ${ }^{2}$, Ashrafun Nahar Monika ${ }^{2}$ \\ ${ }^{1}$ Accelerator Facilities Division, Atomic Energy Center, Dhaka-1000, Bangladesh \\ ${ }^{2}$ Medical Physics Division, Atomic Energy Center, Dhaka-1000, Bangladesh
}

\begin{abstract}
Packed and unpacked spices may contain high level of lead that poses health threats. It may be due to soil contamination or artificial mixing of heavy elements to make the spices look brighter to attract customers. Some spices sample like turmeric and red chilli powder of local market in Bangladesh were collected to determine the toxicity present in them. The sample were dried and pressed to make pellet and irradiated by proton beam of $2.4 \mathrm{MeV}$ and in the current range of $10-15 \mathrm{nA}$ using $3 \mathrm{MV}$ Van de Graaff Acclerator, Atomic Energy Centre, Dhaka and the IBA technique PIXE has been used for all of the experiments. Accuracy and precision of the technique were assured by analyzing certified reference material in the same experimental conditions. The elements that were found to be present in these samples are $P, S, C l, K, C a, M n, F e, Z n$. Among the investigated sample, one turmeric powder sample contains excess Pd which may affect human health. Proper care should be taken on processing the spices.
\end{abstract}

Keywords: PIXE, spices, toxicity.

\section{Introduction}

A spice is a dried seed, fruit, root, bark, or vegetable substance mostly used to improve flavor, color and also to preserve food. Spices grow widely in various regions of the world and have been used for several purposes including medicinal, religious ritual, cosmetics or perfume production, or as a vegetable [1]. Bangladesh has a high diversity of plants used as spices, herbs, and traditional medicines. Several spices are either produced on small farmlands or naturally grow in different regions. But, it is a matter of pity that the use of chemical is one of the main reasons for excessive lead in spices used for cooking for every Bangladeshi family. The chemical is generally used in industry and also are available in the market in the spices producing regions. So there may be some possibility to add these chemical with the spices to improve color for people attraction as well as to traders and spice processing firms. There is often little information available about the safety of those plants and their products in respect to heavy metal contamination. The permissible level of lead in the product is $2.5 \mathrm{ppm}$ in Bangladesh [2]. Due to the significant amount of spices consumed, it is important to know the toxic metal contents in these spices [3]. Lead can accumulate on the body over time and too much of it can cause various health problems, including delayed mental and physical growth and learning deficiencies. It is almost precarious for expecting mothers, infants and young children, according to the Food and Drug Administration (FDA) of the US.

After the detection of High level of lead in Pran's turmeric in Bangladesh by FDA in 2013 [2], some spices sample like turmeric and red chilli powder of local market in Bangladesh were collected to determine the toxicity present in them. The objective of this work is to accurately analyze the levels of toxic heavy metals like lead, cadmium and arsenic that present in some major spice brands and also in open local market available in Bangladesh.

\subsection{Sample Preparation}

\section{Materials And Method}

Samples of Turmeric of different renowned brand and also from local open market were collected in 2014, Dhaka, Bangladesh. Each sample was properly labeled and the weight of each sample was taken and recorded separately. Then the samples were kept on the drying oven ("Memmert Schutzart DIN 40050 - IP 20" at temperature of $60^{\circ} \mathrm{C}$ ) for about eight hour in individual petri-dish. The samples were made dry in such a way that it contains no aqua which was obtained by recording the mass of the sample with a micro balance. Then the samples were grinded with an Agate Mortal Pester into fine powder. Pester, pliers and other related equipment were also cleaned with acetone after grinding each sample in order to avoid the cross contamination. Then the samples were pelletized using Hydraulic Pellet Maker. In this experiment the pellets were made of diameter 0.7 $\mathrm{mm}$ at $120-130 \mathrm{~kg} / \mathrm{cm}^{2}$ pressure. Then the pellets were compressed in holder for radiation in PIXE techniques [4-5]. 


\subsection{Sample Irradiation and Data Acquisition}

The samples were irradiated by proton beam of $2.4 \mathrm{MeV}$ and in the current range of $10-15 \mathrm{nA}$ using 3 MV Van de Graaff Acclerator, Atomic Energy Centre, Dhaka and the IBA technique PIXE has been used for all of the experiments. The emitted X-rays were measured using HPGe X-ray detector and associated data acquisition system. Data were analyzed using GUPIX with DAN32 interfacing software that can simply, automatically and quickly fit the PIXE spectra to obtain the elemental concentrations. Elemental concentration calculation of each element $\mathrm{C}_{Z}$ is based on the following equation [6, 7]:

Where

$$
C_{Z}=\frac{Y}{Y_{t} Q \varepsilon T H}
$$

$Y_{t}=\mathrm{X}$-ray theoretical intensity (i.e. the yield per micro-Coulomb of charge per unit concentration per

steradian),

$Y=\mathrm{X}$-ray experimental intensity or yield

$\mathrm{Q}=$ Measured proton beam charge, $\varepsilon$ is the efficiency of the detector

$\mathrm{T}=$ Transmission through any filter or absorbers between the target and the detector.

$\mathrm{H}=$ Instrumental constant equivalent to the product of the geometrical solid angle of the $\mathrm{x}$-ray detector and any systematic normalization factor present in the charge integration system.

\section{Results and Discussion}

In the present study, five turmeric powder samples and five red chili powder samples were analyzed. The analytical result with \% of errors and Limit of Detection (LOD) are tabulated in Table 1 and Table 2. All the experiment was done at same proton energy of $2.4 \mathrm{MeV}$. The element determined in the varying concentrations includes Phosphorous, Sulpher, Potassium, Calcium, Manganese, Iron, and Zinc. It is clear that Potassium and Calcium are the most abundant detected elements in the samples. Calcium is an essential element for maintaining healthy bones and teeth and also of neuromuscular systemic and cardiac function [8]. Potassium contained in the spices in the range from 1069 to $7708 \mathrm{ppm}$ which is reported to be important for osmotic pressure of the body fluids [9]. The iron contain is fairly good in these spices ranging from 97.7 to $2171 \mathrm{ppm}$. Iron play a vital role in human heaths since it is an essential component of the haemoglobin of the blood and active in the maintaining healthy immune system [10]. Transition elements $\mathrm{Mn}, \mathrm{Fe}, \mathrm{Zn}$ are found at varying concentrations in all the studied samples. These and other essential elements perform various complimentary vital functions in the body to keep the organism healthy. Graphical representation of mean concentration (in ppm) of Turmeric and Red Chili powder is shown in Fig 1. In both cases $\mathrm{K}$ and $\mathrm{Ca}$ are the majority elements where turmeric powder contains much more $\mathrm{K}$ than that of Red chili powder.

Table-1: Elemental concentration (in ppm), \% of Errors and Limit of Detection (LOD) of Turmeric Powder

\begin{tabular}{|c|c|c|c|c|c|c|}
\hline \multicolumn{7}{|c|}{ sample } \\
\hline Sample ID & $\mathbf{P}$ & $\mathbf{S}$ & $\mathbf{K}$ & $\mathbf{C a}$ & Mn & $\mathbf{F e}$ \\
\hline \multirow[t]{3}{*}{$\mathrm{T}-01$} & 611 & 822 & 26822 & 5638 & 2267 & 898 \\
\hline & $2.55 \%$ & $1.94 \%$ & $0.2 \%$ & $4.12 \%$ & $1.5 \%$ & $4.2 \%$ \\
\hline & 19.5 & 17.3 & 21.0 & 328 & 31.8 & 66.6 \\
\hline \multirow[t]{3}{*}{$\mathrm{T}-02$} & 641 & 966 & 51331 & 7208 & 438 & 2171 \\
\hline & $3.33 \%$ & $2.5 \%$ & $0.18 \%$ & $6.3 \%$ & $14.5 \%$ & $3.8 \%$ \\
\hline & 28.8 & 27.2 & 35.6 & 645 & 114 & 136 \\
\hline \multirow[t]{3}{*}{ T-03 } & 598 & 899 & 44679 & 5802 & 973 & 1110 \\
\hline & $2.9 \%$ & $2.2 \%$ & $0.18 \%$ & $7.4 \%$ & $4.4 \%$ & $4.7 \%$ \\
\hline & 22.4 & 19.1 & 30.8 & 612 & 66.5 & 85 \\
\hline \multirow[t]{3}{*}{ T-04 } & 700 & 1018 & 48173 & 6705 & 827 & 1089 \\
\hline & $2.9 \%$ & $2.4 \%$ & $0.19 \%$ & $7.2 \%$ & $6.17 \%$ & $5.8 \%$ \\
\hline & 26.1 & 25.4 & 39.3 & 668 & 83.1 & 108.1 \\
\hline \multirow{3}{*}{ T-05 } & 650 & 850 & 45800 & 6053 & 1126 & 1256 \\
\hline & $5.8 \%$ & $2.6 \%$ & $0.2 \%$ & $5.2 \%$ & $5.2 \%$ & $4.5 \%$ \\
\hline & 20 & 18.4 & 33.5 & 456 & 70.6 & 50.3 \\
\hline
\end{tabular}

Table-2: Elemental concentration (in ppm), \% of Errors and Limit of Detection (LOD) of Red Chili Powder sample (N= Below Limit of Detection)

\begin{tabular}{|c|c|c|c|c|c|c|c|}
\hline Sample ID & $\mathbf{P}$ & S & $\mathbf{K}$ & $\mathbf{C a}$ & Mn & $\mathbf{F e}$ & Zn \\
\hline \multirow[t]{3}{*}{ C-01 } & 221 & 335 & 6337 & 1843 & \multirow[t]{3}{*}{$\mathrm{N}$} & 1410 & \multirow[t]{3}{*}{$\mathrm{N}$} \\
\hline & $4.1 \%$ & $3.1 \%$ & $0.3 \%$ & $4.4 \%$ & & $1.7 \%$ & \\
\hline & 12.3 & 12 & 8.5 & 129.6 & & 26.2 & \\
\hline \multirow[t]{2}{*}{$\mathrm{C}-02$} & 309.6 & 473 & 7708 & 2546 & 73.8 & 992.3 & 63.3 \\
\hline & $3.2 \%$ & $2.2 \%$ & $0.3 \%$ & $4.5 \%$ & $24.8 \%$ & $2.42 \%$ & $31.6 \%$ \\
\hline
\end{tabular}




\begin{tabular}{|c|c|c|c|c|c|c|c|}
\hline & 12.8 & 11.5 & 7.5 & 173 & 33 & 31.6 & 34.2 \\
\hline \multirow[t]{3}{*}{ C -03 } & 275 & 542 & 1069 & 2258 & 33.7 & 705 & 136.2 \\
\hline & $3.7 \%$ & $2.3 \%$ & $0.3 \%$ & $4.5 \%$ & $35.72 \%$ & $3.1 \%$ & $14.2 \%$ \\
\hline & 13.1 & 13.4 & 10.9 & 150.9 & 21.1 & 20.8 & 21.8 \\
\hline \multirow[t]{3}{*}{ C -04 } & 93.8 & 239 & 2875 & 656.6 & \multirow[t]{3}{*}{$\mathrm{N}$} & 97.7 & 43.7 \\
\hline & $6.1 \%$ & $2.7 \%$ & $0.6 \%$ & $5.13 \%$ & & $9.6 \%$ & $26.4 \%$ \\
\hline & 7.4 & 7 & 6.4 & 51 & & 9.6 & 13.1 \\
\hline \multirow[t]{3}{*}{ C -05 } & 194 & 387 & 6651 & 1403 & 41.2 & 406.8 & 87.9 \\
\hline & $4.1 \%$ & $2.4 \%$ & $0.4 \%$ & $4.8 \%$ & $19 \%$ & $3.8 \%$ & $16 \%$ \\
\hline & 10.4 & 9.7 & 13.4 & 100.3 & 9.6 & 12.8 & 18.9 \\
\hline
\end{tabular}

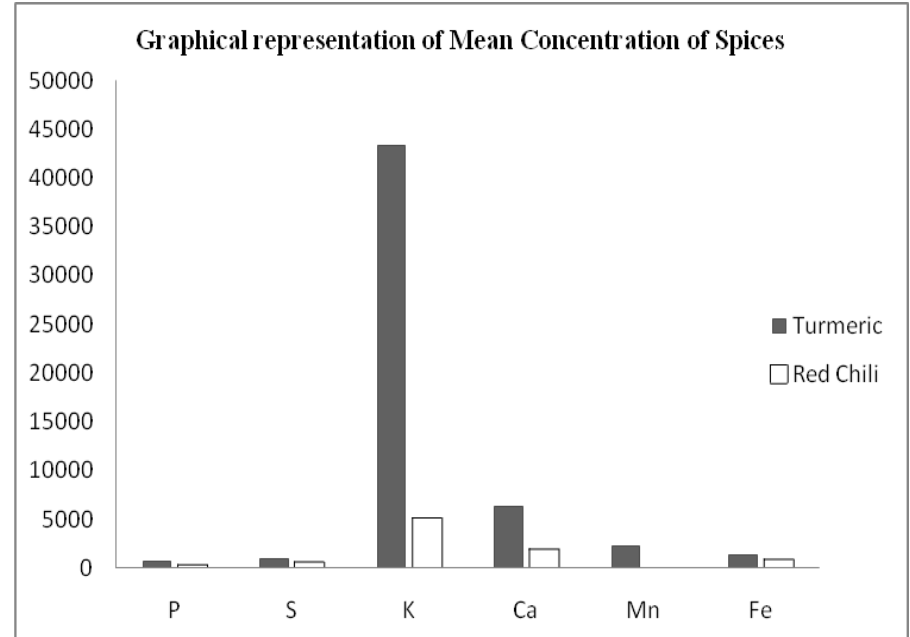

Figure 1: Graphical representation of mean concentration (in ppm) of Turmeric and Red Chili powder

In the present study, only one Turmeric sample (T-3) contains lead with $708 \mathrm{ppm}$ which is above the permissible level and this sample also has chromium concentration (70 ppm, $44 \%$ of Error, and LOD- 55) above the limit of detection. The lead enters into turmeric from 27 various sources, beginning from the soil. Soil in some regions contains high level of lead. Lead also takes place while cleaning turmeric using alum [3]. Most of the spices processing companies' bye turmeric from traders as they are yet to develop direct contact with the farmers and in the absence of proper quality checks by the companies, the trader may supplies the big firms with contaminated turmeric.

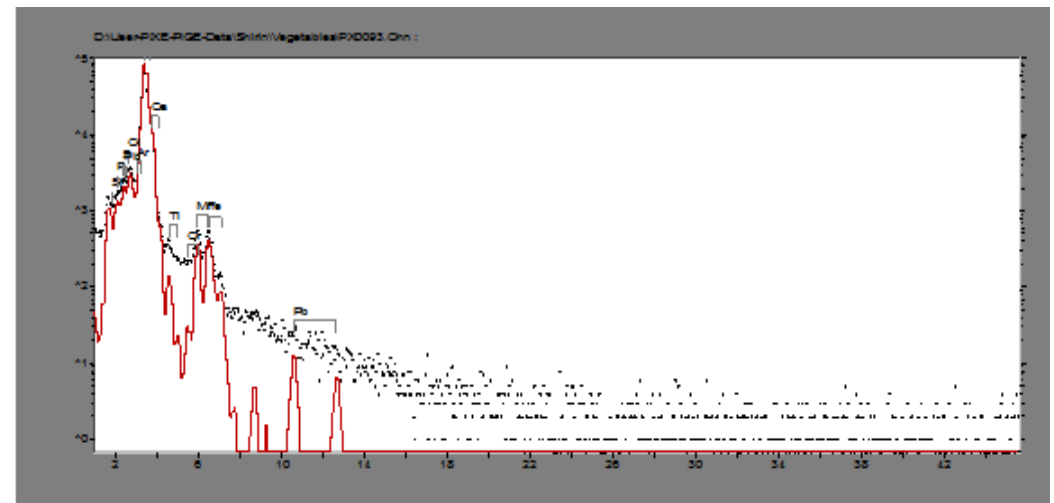

Figure 2: Elemental Concentration in ppm of Turmeric powder sample (T-03) where $\mathrm{X}$-axis represents energy in $\mathrm{KeV}$ and $\mathrm{Y}$-axis represents counts per second

Comparison of the lead constituents of spices samples in ppm of different studies with that of present work are as follow:

\begin{tabular}{|c|c|}
\hline $\begin{array}{c}\text { Lead in turmeric powder sample } \\
\text { (Concentration in ppm) }\end{array}$ & Reference Study \\
\hline $708 \mathrm{ppm}$ & Present study \\
\hline $182 \mathrm{ppm}$ & {$[3]$} \\
\hline$(80-483) \mathrm{ppm}$ & {$[12]$} \\
\hline
\end{tabular}




\section{Conclusion}

Turmeric and red chili powder are spices commonly used by Bangladeshi people for cooking purpose. Proton Induced X-ray Emission technique has been employed to examine spices samples to monitor the levels of toxic heavy metals like lead, cadmium and arsenic present in that sample. Lead poisoning remains a major public health problem, particularly for young children in developing countries. Uptake of soil elements into the turmeric may be possible but the maximum uptake of lead into the root of the plant to be approximately $10 \%$ [11]. The addition of lead chromate to the sample (to increase the weight and bright color) may also be the source of lead contamination. There is an urgent need to identify the source of lead exposure in rural communities in Bangladesh. Although one sample contains lead above the permissible level, the method of contamination is still unidentified. Therefore, further study is needed to confirm these sources.

\section{Acknowledgements}

The author would like to thank at the staff of Accelerator Facilities Division, Atomic Energy Centre Dhaka, Bangladesh for giving technical support in this work.

\section{References}

[1]. H. Mubeen, I. Naeem, A. Taskeen and J. Saddiq, New York Science Journal, 2(5), 2009, 1554-0200.

[2]. The Daily Star, Bangladesh, March 08, 2014.

[3]. R. P. Choudhury and A. N. Garg. Food Chemistry, 104 (2007) 1454-1463.

[4]. S. Akter, M. M. Ahasan, M. J.Abedin, R. Khatun, A. N. Monika "Elemental Profile Studies of some Soil Samples using Particle Induced X-Ray Emission Technique", International Journal of Reciprocal Symmetry and Theoretical Physics, 1(2), $2014,106-110$.

[5]. M. L. Hossen, S. M. A. Islam, M. J. Abedin, S. Akter, O. F. Rasel, M. M. Ahasan, R. Khatun, A. N. Monika, "Elemental Profile Analysis of Some Traditional Medicinal Plants of Bangladesh Using PIXE Technique", Journal of Nuclear and Particle Physics, 4(5), 2014,137-141.

[6]. S. A. E. Johnson and J. L. Campbell, PIXE: A Nobel Technique for elemental Analysis (New York, John Willy \& Sons, 1988)

[7]. J. L. Campbel, D. Higuchi, J. A. Maxwell, W. J. Teesdale, "Quantitative PIXE microanalysis of thick specimen", (Nucl. Instr. and Meth, B 77, 1993)

[8]. C. D. Berdanier, Advanced Nutririon-Micronutrients (CRC Press, New York, 1994).

[9]. G. Kolata, Science 216 (1982), 38.

[10]. C. V. Broome, J. S. Marks, Recommendations to prevent and control iron deficiency in the United States in B. R. Holloway, A. G. Dean (Eds.), Morbidity and Mortality weekly report, US Department of Health and Human services, Atlanta, Georgia, 1998.

[11]. S. A. Rahim, W. M. R. Idris, Z. A. RAhman, T. Lihan, R. Omar, and L. K. Yan, "Heavy metal content in selected flavoring plants and in ultra-basis soil of Felda Rokan Barat, Negeri Sembilan, Malaysia", Sains Malaysisn, 41(1), pp. 11-21, 2012.

[12]. K. Gleadon et. al., "Contaminated Turmeric is a potential source of lead exposure for children in rural Bangladesh", Journal of Environmental and Public Health, 2014, pp. 1-5. 